\title{
Einstein and Lorentz
}

SIR - Michaël W. J. van den Brink recalls a sombre occasion when Einstein acknowledged his profound scientific debt to $\mathrm{H}$. A. Lorentz ${ }^{1}$.

When, in 1920, Lorentz snubbed Max Planck's sixtieth birthday celebration in solidarity with ascendant post-1918 antiGerman revanchism, he was rebuked by Max Born for his blindness to Lorentz's primitivism $^{2}$. Indeed, in 1944, Einstein echoed Lorentz's absolute anti-Germanism when he wrote: "The Germans as an entire people are responsible for these mass murders and must be punished as a people..." ${ }^{3}$ Einstein still felt the same way in 1953; again, the usually deferential Born was compelled to remonstrate in a reply to a letter from Einstein: "I only want to tell you that the German Quakers have their headquarters in Pymont. They are no 'mass-murderers', and many of our friends there suffered far worse things under the Nazis than you or I. One should be chary of applying epithets of this sort. The Americans have demonstrated in Dresden, Hiroshima and Nagasaki that in sheer speed of extermination they surpass even the Nazis." 2

But Einstein's grandest and purest genuflection to Lorentz came in 1954: "In January 1954 Einstein was asked: Who were the greatest men, the most powerful thinkers whom he had ever known? He answered without hesitation, 'Lorentz'." Then he added: "I never met Willard Gibbs; perhaps, had I done so, I might have placed him beside Lorentz." 4

William Steinsmith

1239 Castenada Avenue,

San Francisco,

California 94116, USA

SIR - Einstein had acknowledged his debt to Maxwell and Lorentz unequivocally in his address of 15 May 1940 to the 8th American Scientific Congress in Washington, DC. An abridged version was published in Nature a month later ${ }^{5}$. Einstein said:

The theory of relativity arose out of efforts to improve, with reference to logical economy, the foundation of physics as it existed at the turn of the century. The socalled special or restricted relativity theory is based on the fact that Maxwell's equations (and thus the law of propagation of light in empty space) are converted into equations of the same form, when they undergo Lorentz transformation. This formal property of the Maxwell equations is supplemented by our fairly secure empirical knowledge that the laws of physics are the same with respect to all inertial systems. This leads to the result that the Lorentz transformation - applied to space and time coordinates - must govern the transition from one inertial system to any other. The content of the restricted relativity theory can accordingly be summarized in one sentence: all natural laws must be so conditioned that they are co-variant with respect to Lorentz transformation.

Einstein was not without faults, but non-attribution of credit to Maxwell and Lorentz for his ideas on relativity is not one of them.

\section{Sachi Sri Kantha}

5-16-305 Tsukimicho,

Fukuroi City,

Shizuoka 437-01,

Japan

1. van den Brink, M. W. J. Nature 375, 272 (1995)

2. Born-Einstein Letters (Macmillan, London, 1971).

3. Einstein, A. Ideas and Opinions, 212.

4. Mehra, J. Physica 79A, 474 (1995).

5. Nature 145, 920-924 (1940).

\section{Lucifer principle}

SIR - In The Lucifer Principle, Howard Bloom attempts to write a world history from a modern scientific viewpoint, but your reviewer seems to have mistaken it for an evolutionary tract whose main purpose is to defend group selection against individual selection. Only a few paragraphs were in fact devoted to that topic, and the main part of the book is about the past few thousand years of human history. For this, it does not matter whether group selection is a common occurrence or a very rare one.

What Bloom draws attention to is the firm grip that human culture holds on the individual human mind, and the role this relationship has played in the horrors and triumphs of human history. Human social culture is radically different from that of other animals because it develops by acquiring new knowledge and incorporating it for transmission to succeeding generations; this process is of course orders of magnitude faster than Darwinian evolution. The development of human culture, and the human individual's almost total (though seldom self-acknowledged) submission to it, have been hugely important in giving us a dominant position on this planet, where we pose a serious threat to many of its other inhabitants. Though it is only a single episode, and no one knows how it will end, does not the human population explosion constitute a proof that group selection can occur?

Your reviewer (Mark Pagel, in Nature $374,828 ; 1995)$ is a member of the BBSRC-NERC Ecology and Behaviour Group in Oxford, which must be vitally concerned with the reasons for humanity's competitive success; it is therefore curious that he should dismiss with a genteel cry of "déjà $v u$ " the main part of Bloom's message.

\section{Horace Barlow}

Trinity College,

Cambridge CB2 3EG, UK

\section{Free exchange}

SIR - In response to your article on the French genome programme (Nature 375, $175 ; 1995)$, we should like to point out, on behalf of the members of the Washington University Genome Sequencing Center and the Sanger Centre, that our respective laboratories have always freely and promptly released their data without restrictions of any kind, and will continue to do so in the future. As far as we are concerned, there is therefore no question of France being in a "position of dependence" as the French report you refer to claims.

Furthermore, we have explicitly put forward the human sequencing project as a subject for international cooperation (see, for example, Nature 375, 93; 1995). We intend to do our bit, but the project can be completed only if others will do theirs. With the map coming to fruition, the human genome can readily be divided by chromosome or by region for the sequencing task, just as the yeast genome was successfully divided for the same purpose. If all the national programmes contribute, the community can have the human genome sequence in hand by the year 2001 . Bob Waterston

John Sulston

The Sanger Centre, Hinxton Hall, Hinxton, Cambridge CB1O 1RQ, UK

\section{Remarkable delay}

SIR - I was surprised to read the "Corrigendum" in Nature $(\mathbf{3 7 5}, 88 ; 1995)$ to a paper published many months ago (T. D. White et al. 371, 306-312; 1994). Even if the correction was to remedy a serious typographical error or other inadvertent mistake, the delay would be remarkable; but it is clear from the Corrigendum that the error is not inadvertent as it seeks to create a new genus in the Superfamily Hominoidea on the grounds that new finds made subsequent to the initial publication render the original conclusions unacceptable to the authors. The new finds are alluded to and a diagnosis is given in the Corrigendum, but the material is neither described nor illustrated.

It is, of course, the right of the authors to alter their views on the classification of their materials, but it is also their duty to make available the evidence for these changes. This duty will no doubt be fulfilled in due course. There may be some, however, who will feel that the hasty erection of a new genus in this way has more to do with expediency and priority than with scientific enlightenment. Others may feel that the editor has been bowled a fast straight one that took out his middle stump!

M. H. Day

Natural History Museum,

Cromwell Road, London SW7 5BD, UK 